\title{
Scale-Bridging 3D Analysis of Micro-/Macroporous Zeolite Particles Using X-Ray Nano-Tomography and Electron Tomography
}

\author{
Benjamin Apeleo Zubiri ${ }^{1 *}$, Tobias Weissenberger ${ }^{2}$, Thomas Przybilla ${ }^{1}$, Janis Wirth ${ }^{1}$, Silvan Englisch ${ }^{1}$, \\ Dominik Drobek $^{1}$, Wilhelm Schwieger ${ }^{2}$ and Erdmann Spiecker ${ }^{*}$ \\ 1. Institute of Micro- and Nanostructure Research (IMN) \& Center for Nanoanalysis and Electron \\ Microscopy (CENEM), University of Erlangen-Nuremberg, Erlangen, Germany. \\ 2. Chair of Chemical Reaction Engineering, University of Erlangen-Nuremberg, Erlangen, Germany. \\ * Corresponding authors: benjamin.apeleo.zubiri@fau.de, erdmann.spiecker@fau.de
}

Material properties are often governed by morphologies on certain length scales which can be addressed by different 3D imaging techniques providing complementary advantages. Nanoparticulate agglomerates or macroporous particles in the lower $\mu \mathrm{m}$-range are such examples and two appropriate methods for their 3D exploration are electron tomography (ET) and high-resolution X-ray tomography (Nano-CT). Whereas ET reaches spatial resolutions down to $(1 \mathrm{~nm})^{3}$ of volumes up to $(3 \mu \mathrm{m})^{3}$, the resolution of laboratory Nano-CT can get down to $(50 \mathrm{~nm})^{3}$ with samples sizes of up to $(64 \mu \mathrm{m})^{3}$. So, each technique has its benefits and there is always a balance between spatial resolution and sample size.

Microporous materials such as zeolites are widely used as heterogeneous catalysts. However, the slow transport of reactants to the active sites confined in the micropores is a common problem. Hierarchically structured catalysts consisting of at least two interconnected pore systems, a microporous and an additional larger pore system, could already demonstrate their improved mass transport properties [1]. One system that fulfills these requirements are microporous zeolite crystals with additional intracrystalline macropores [2]. These zeolite crystals (Fig. 1a,b) are synthesized by a steam-assistedcrystallization (SAC) process, where mesoporous silica spheres (MSPs) serve as a sacrificial macropore template and are inversely transformed into the micro-/macroporous MFI-type zeolite. To understand and visualize the morphological changes during the SAC process, the porous particles at different stages of their crystallization process are investigated using ET (Fig. 1, 2) and Nano-CT (Fig. 3).

The samples are prepared using a novel particle transfer method [3], where individually selected particles are transferred from a suitable sample grid onto the plateau of a tomography tip, tailored to the size of the chosen particle, inside a scanning electron microscope (SEM). The samples can be equally used for $360^{\circ}$ ET (Figs 1, 2) and Nano-CT (Fig. 3). $360^{\circ}$ ET experiments were conducted using a Fischione model 2050 on-axis rotation tomography holder, which allows for the acquisition of complete tilt series of projections in a tilt-angle range of at least $180^{\circ}$, inside a FEI Titan Themis 300 transmission electron microscope with an acceleration voltage of $300 \mathrm{kV}$ in high-angle annular dark-field (HAADF) scanning transmission electron microscopy (STEM) mode. A Zeiss Xradia 810 Ultra X-ray microscope with a $5.4 \mathrm{keV} \mathrm{Cr}$ source in phase contrast mode was used for the Nano-CT investigations. The $360^{\circ} \mathrm{ET}$ reconstructions reveal morphological details at higher resolution like smaller internal pores, pore connections or incompletely converted MSPs during a transition state of the final products (Fig. 2c). Even important quantitative measures to describe the pore space like pore size distribution can directly be extracted. In contrast, Nano-CT enables the 3D reconstruction of several particles and particle agglomerates in one measurement, which is important to get statistically relevant data and to be able to investigate the interparticle pore space. Both techniques together allow for a complementary and scalebridging $3 \mathrm{D}$ analysis of the SAC process of these micro-/macroporous zeolite particles, and serves as a 
general model for detailed 3D analyses on those length scales [4].

References:

[1] D Schneider et al., Chemical Society Reviews 45 (12) (2016), p. 3439.

[2] A Machoke et al., Advanced Materials 27 (2015), p. 1066.

[3] T Przybilla et al., Small Methods 2 (2018), p. 1700276.

[4] We gratefully acknowledge the German Research Foundation (DFG) for funding through the priority program SPP 1570, the research training group GRK1896, the project SP648/8 and the DFG EXC 315.
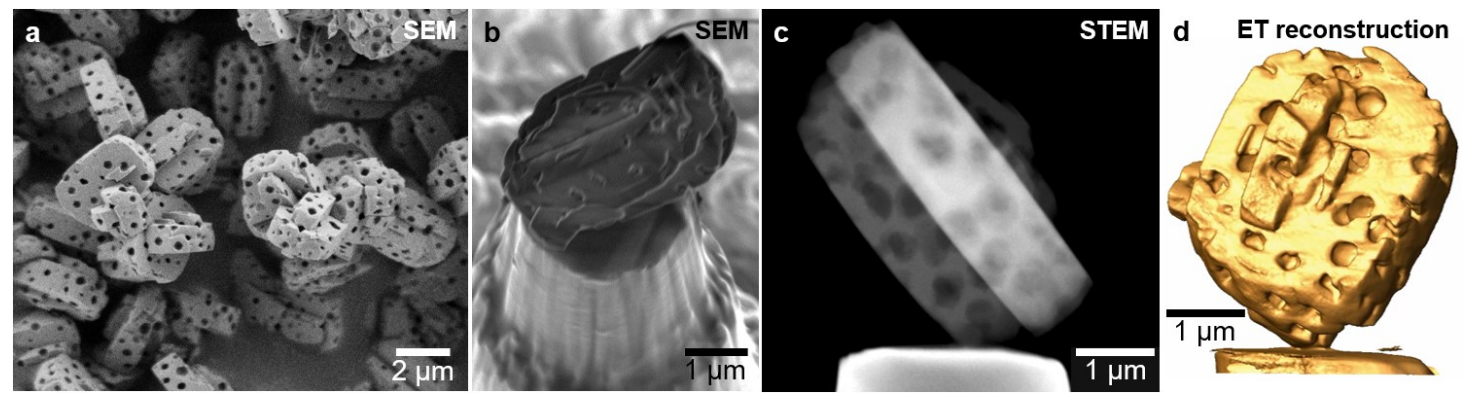

Figure 1. (a) SEM overview image of completely crystallized macroporous zeolite particles. (b) SEM image of one exemplary particle on a tailored tomography tip after particle transfer. (c) HAADF STEM image from the $360^{\circ}$ ET tilt series of the same particle. (d) Surface rendering of the $3 \mathrm{D}$ reconstruction.

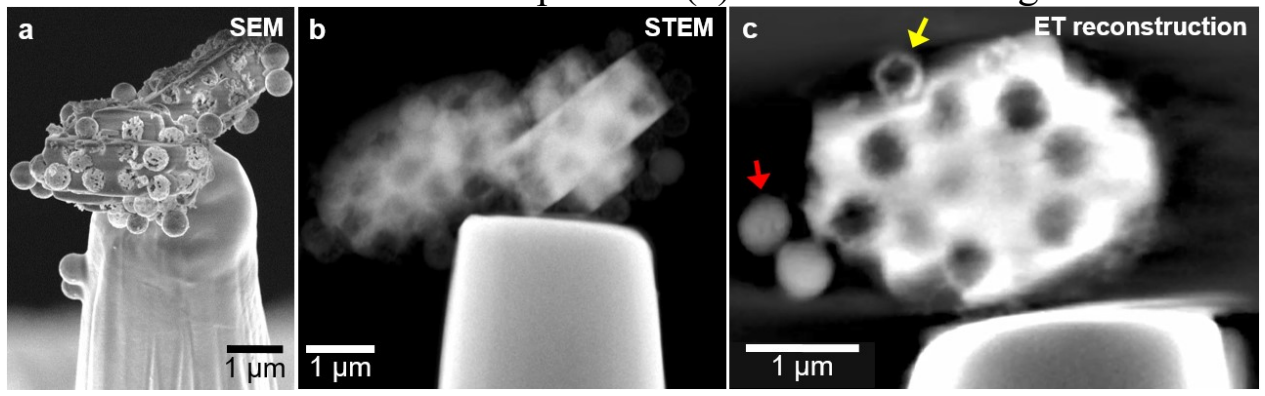

Figure 2. (a) SEM image of two exemplary macroporous zeolite particles in a transient state of the SAC process on a tailored tomography tip. (b) HAADF STEM image from the $360^{\circ}$ ET tilt series of the same particles. (c) Slice through the 3D reconstruction showing the internal macropores and also the proceeding dissolution of the MSP primary particles - some particles appear relatively intact (indicated with red arrow), while other particles are almost completely dissolved and used for the growth of the zeolite crystal (remaining MSP shell indicated with yellow arrow).
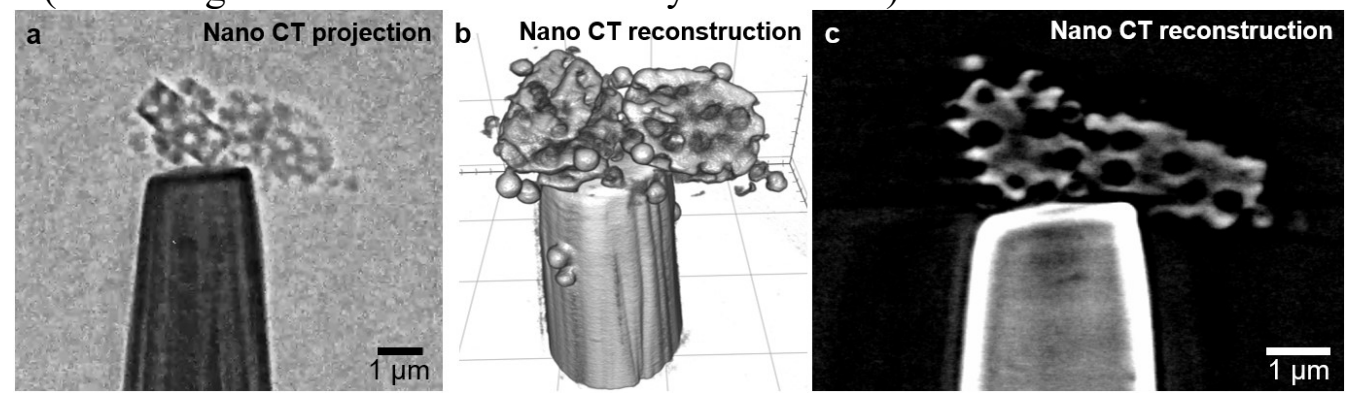

Figure 3. (a) Nano-CT image from the tilt series in phase contrast mode of the same particles as in Figure 2. (b) Volume rendering of and (c) slice through the Nano-CT reconstruction. 